\title{
Real-time manipulation of intestinal peristalsis by enteric-encapsulated magnetic nanoparticles \& wearable 3D-printed devices
}

\author{
Fen $\mathrm{Yu}^{1}$, Xiao Cui ${ }^{2}$, Yuanyuan Lang ${ }^{3}$, Fei Huang ${ }^{4}$, Lingfang Wang ${ }^{4}$, Xinxin Miao ${ }^{3}$, Fanrong $\mathrm{Ai}^{4}$, Caifeng Xie ${ }^{4}$, \\ Hongbo Xin ${ }^{4}$, Cheng Yang ${ }^{5}$ and Xiaolei Wang (1) ${ }^{1,4}$
}

\begin{abstract}
The present study introduced a new biomedical engineering concept to realize the real-time adjustment of nutrient absorption. With the aid of a 3D-printed wearable external magnet device, both the location and vibration of entericcoated magnetic nanoparticles (EMNPs) in the small intestine could be controlled directly. Through a facile mobile app, gastrointestinal motility could be monitored directly in real time. Further tests revealed that this technology could be an attractive way to adjust one's weight without diet or exercise modification.
\end{abstract}

\section{Introduction}

Obesity and metabolic syndrome are a series of worldwide health problems ${ }^{1}$. In 2015, Drs. Colditz and Yang's group reported that $39.96 \%$ of men and $29.74 \%$ of women were overweight and that $35.04 \%$ of men and $36.84 \%$ of women were obese in the U.S ${ }^{2}$. Obesity is a high risk factor for several chronic diseases ${ }^{1}$, such as type 2 diabetes mellitus $^{3-5}$, dyslipidemia, hypertension ${ }^{6}$, coronary heart disease $^{7}$, stroke $^{8}$, and cancer ${ }^{9}$. In addition to avoiding the risk of diseases, women, especially actresses, are always looking to lose weight to keep up a fantasy facade. At present, the main strategies for losing weight include dietary therapy, physical activity, behavior therapy, pharmacotherapy and surgery ${ }^{10}$. Although the first three options are relatively healthier, they are time consuming and strenuous. Generally, pharmacotherapy is the most convenient option, but it is usually accompanied by some

\footnotetext{
Correspondence: Xiaolei Wang (wangxiaolei@ncu.edu.cn)

${ }^{1}$ College of Chemistry, Nanchang University, Nanchang, 330088 Jiangxi, P. R. China

${ }^{2}$ Department of Radiation and Medical Oncology, Zhongnan Hospital, Wuhan University, Wuhan, 430071 Hubei, P. R. China

Full list of author information is available at the end of the article.

These authors contributed equally: Fen Yu, Xiao Cui
}

side effects. For example, the lipase inhibitor orlistat, the most widely used weight-loss drug, has some adverse effects, including fatty stool, fecal urgency, and oily spotting $^{11}$. In addition to pharmacotherapy, bariatric surgery ${ }^{12}$, especially gastric bypass surgery ${ }^{13}$, is currently one of the most efficient protocols ${ }^{14}$. However, this strategy also has its own complications, including incisional hernia and vitamin B12 deficiency ${ }^{15}$. Moreover, because surgery is irreversible, the side effects caused by gastric bypass surgery are usually unrecoverable for the patients.

Fundamentally speaking, as shown in Scheme 1a, the reason for gaining weight is not complicated. In simple terms, obesity results when people consume more energy than they expend. However, the conventional way to lose weight (such as diet, exercise, or even gastric band operation) would inevitably disrupt one's regular daily routine, which could adversely impact the quality of life. Indeed, the most ideal protocol is to adjust one's weight without surgery, dietary changes or extra exercise. That is, by reducing the absorption ratio, weight loss could be realized without diet or exercise (consumption) modification, even if intake is greater than consumption (Scheme 1b). In theory, this attractive protocol is not, in 

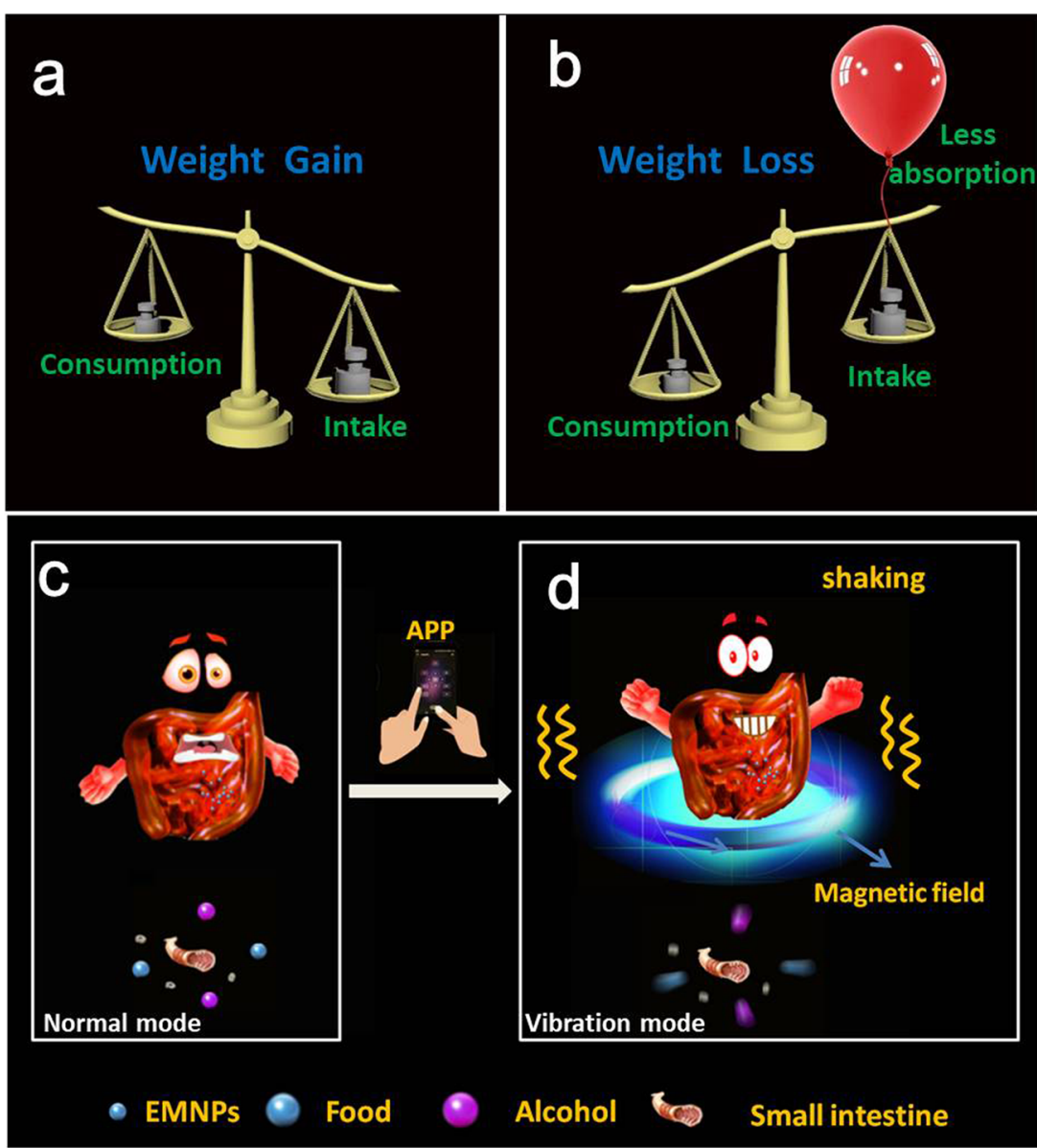

Scheme 1 Illustration of the proposed technical concept. a Schematic diagram of weight gain. When the intake is greater than consumption, the body weight increases. $\mathbf{b}$ Theoretical basis of the present study. By reducing the absorption rate, we can achieve weight loss without dieting or exercising. $\mathbf{c}$, d Schematic of intestinal peristalsis stimulated by the vibration of EMNPs. The small intestine would accelerate peristalsis under the stimulation of EMNPs with a magnetic field and regulate the absorption of food and alcohol. The strength of the H-field can be controlled by a mobile app, which can adjust the absorption ratio of the intake

fact, impossible if the absorption ratio of intake nutrients could be precisely controlled. The small intestine is the major organ of nutrient digestion and absorption, and small intestine peristalsis is directly related to its absorption efficiency ${ }^{16,17}$. Based on the above discussion, a novel weight-loss concept that combines the advantages of bariatric surgery (effective) and pharmacotherapy (convenient) was proposed in this study. The most innovative part of this study was the introduction of 3Dprinted wearable devices to provide tunable vibrating magnetic power that led to mechanical stimulation, which enhanced intestinal peristalsis and thus minimized the absorption of intestinal villus. To achieve this target, enteric-coated magnetic nanoparticles (EMNPs) were synthesized for oral administration because both the enteric capsule (E-capsule ${ }^{18}$ and magnetic nanoparticles $(\mathrm{MNPs})^{19}$ had been approved by the FDA (Food and Drug Administration $)^{20}$. With the aid of an external magnetic field generated by a wearable magnetic device, the EMNPs could be located and vibrated in the upper intestine where the majority of nutrients were absorbed ${ }^{21}$. Alcohol abuse is a well-known worldwide problem that jeopardizes human health ${ }^{22}$, and alcohol absorption occurs in the upper intestine as well. In this sense, the EMNPs will also help to reduce drunkenness through alcohol detoxification, which stimulates intestinal peristalsis and accelerates the excretion of alcohol.

\section{Materials and methods Fabrication of EMNPs}

A total of $1.5 \mathrm{~g}$ enteric capsules was added to $10 \mathrm{ml}$ of a weakly alkaline solution. After the capsules were completely dissolved, $0.01 \mathrm{~g}$ magnetic nanoparticles was added. Then, the magnetic nanoparticles were completely encapsulated by stirring. Finally, enteric magnetic nanoparticles (EMNPs) could be obtained. 

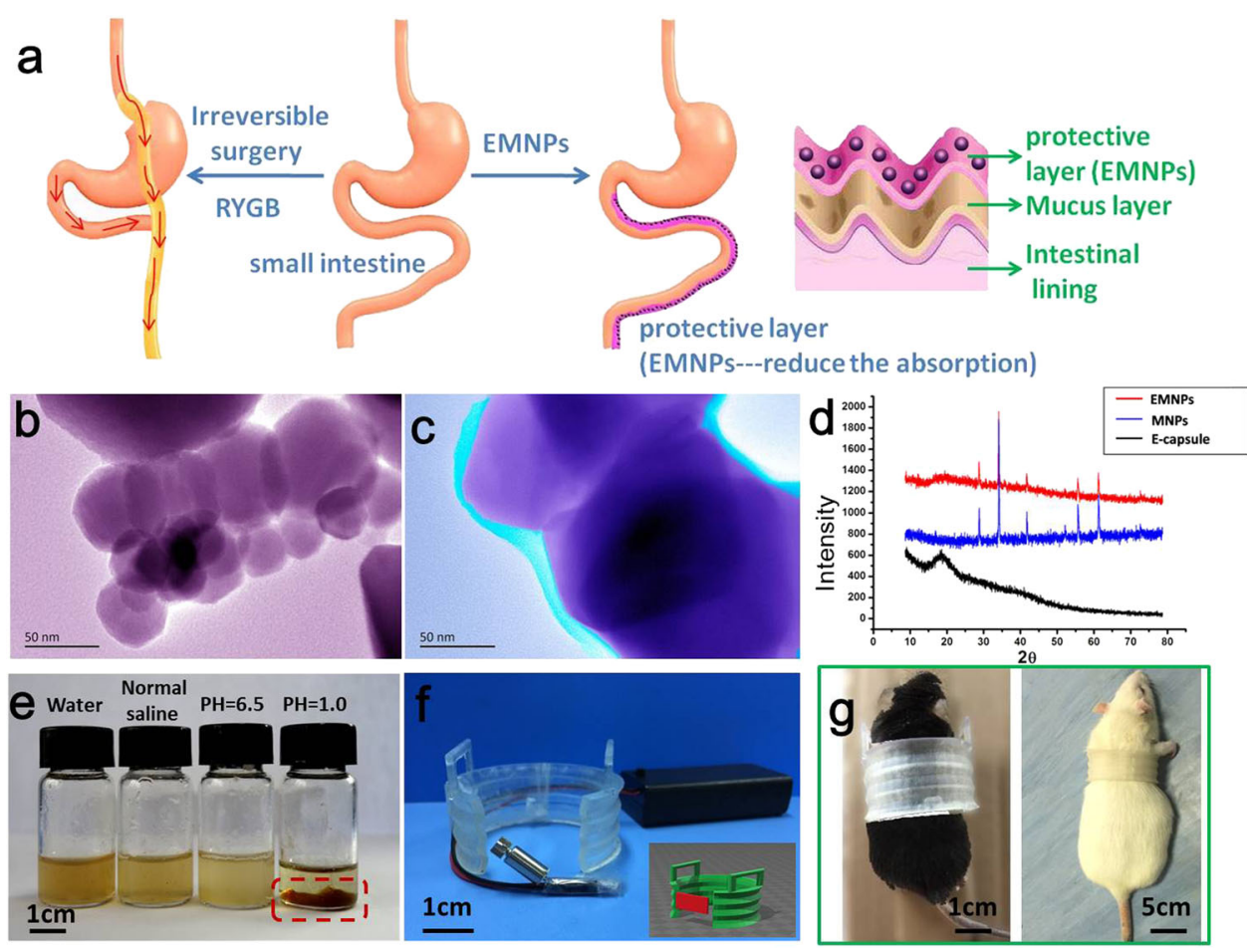

Fig. 1 The characterization of EMNPs' structure. a Illustration of how EMNPs could be used as a new method to replace irreversible bariatric surgery (RYGB). $\mathbf{b}$ TEM image of MNPs. c TEM image of EMNPs. The scale bar is $50 \mathrm{~nm}$. The blue part represents the membrane of the enteric capsule, and the purple part represents the MNPs. $\mathbf{d}$ XRD data of the EMNPs, MNPs and E-capsule (enteric capsule). e Reaction of the enteric capsule in different solutions (water, saline, $\mathrm{pH}=6.5$ (simulated intestinal fluid) and $\mathrm{pH}=1.0$ (simulated gastric fluid)) to form water-miscible liquids; all except simulated gastric fluid. $\mathbf{f}$ Optical diagram of a 3D-printed elastic device with an $\mathrm{H}$-field. The inset shows a customized wearable elastic device model, which was digitally designed for 3D-printing techniques. $\mathbf{g}$ Optical photographs of C57BL/6 mice (left) and SD rats (right) with the wearable weightloss device

\section{Continuous medication and intermittent medication of C57BL/6 mice}

Each mouse was treated with $0.1 \mathrm{ml} / 10 \mathrm{~g}$ EMNPs a day for three weeks, weighed on each weekend, and phlebotomized and tested for the TC, TG and D-xylose of blood with a reagent kit on the third weekend. Treatment was stopped for one week during the fourth week, and the mice were weighed and phlebotomized for the blood content test. Then, the mice were medicated again for one week and weighed; treatment was stopped at the sixth week, and the weight of the mice was recorded on the sixth weekend.

\section{Results}

\section{Characterization of EMNPs}

In this study, the regulation of intestinal peristalsis was divided into three steps. First, the enteric capsule was used to wrap the magnetic nanoparticles, which could not only protect the EMNPs from erosion by gastric acid but also cover the epithelium of the small intestine and then form a barrier to block absorption. Afterwards, these capsules would be gradually dissolved by intestinal fluids, thereby releasing the magnetic nanoparticles. Subsequently, with the aid of 3D-printing technology, a wearable external magnetic field device could be customized according to different populations. Finally, by manipulating the mobile app, the magnetic field could be remotely controlled as required and would realize the real-time regulation of food and alcohol absorption to achieve the goal of weight control (Scheme 1c, d).

The enteric capsule was used as a package, and its main components were HPMC (hydroxyl propyl methylcellulose) and HPMCP (hydroxyl propyl methylcellulose phthalate $)^{23}$. The IR spectrum of the enteric capsule is provided in Fig. S1. The EMNPs were designed to form a physical barrier on the mucosa against substances such as nutrients, acids and digestive enzymes, while the magnetic nanoparticles could promote gastrointestinal peristalsis under magnetic fields (Fig. 1a). As shown in Fig. 1b, the size of a typical MNP was approximately $50 \mathrm{~nm}$, and the original TEM (transmission electron microscopy) images are presented in Fig. S2. An enteric capsule membrane that successfully covered the MNPs could be observed by TEM (Fig. 1c) ${ }^{24}$, which agreed with the X-ray diffraction (XRD) spectrum (Fig. 1d). However, all enteric capsules were used as protective membranes for the MNPs to 

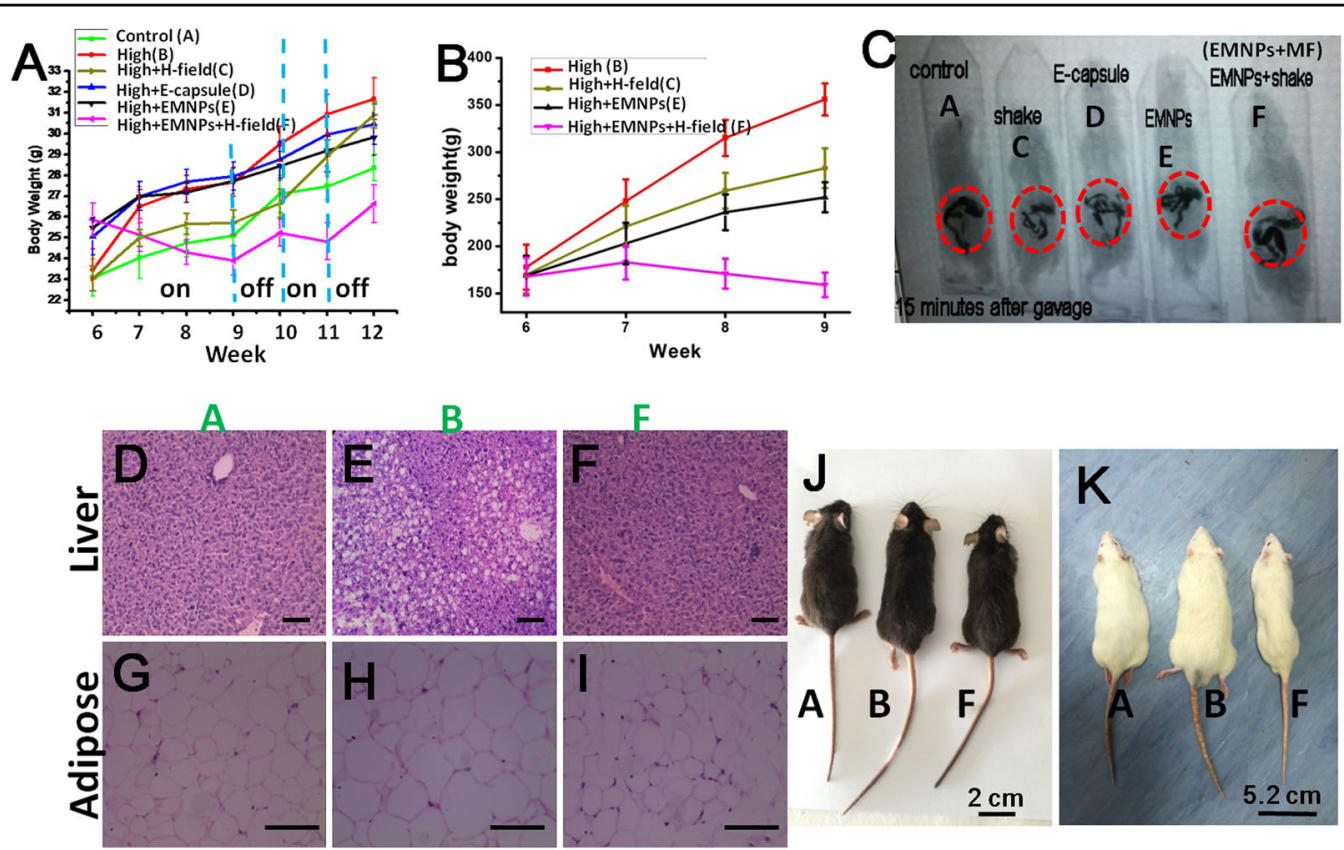

Fig. 2 Effect of different treatments to mice. $\mathbf{a}$, $\mathbf{b}$ The body weights of the C57BL/6 mice and SD rats were variable with different treatments. Group A: fed with normal food and without other treatment; group B: fed with high-fat food and without other treatment; group C: fed with high-fat food and with a varying magnetic field (MF); group D: fed with high-fat food and treated with E-capsules; group E: fed with high-fat food and treated with EMNPs; group F: fed with high-fat food and treated with both EMNPs and varying magnetic field. "On" represents continuous medication; "off" represents that the treatment was withdrawn. $\mathbf{c}$ Digital gastrointestinal contrast radiography of mice with different treatments. $\mathbf{d}-\mathbf{f}$ Images of hematoxylin eosin (HE) staining of liver tissue slices for three groups, namely, group A, group B and high group F, respectively. $\mathbf{g}$-i Images of hematoxylin eosin (HE) staining of tissue slices of adipose tissue for group A, group B and high group F, respectively. j, $\mathbf{k}$ Optical images of the body forms of the C57BL/6 mice and SD rats after different treatments (group A, group B and group F). Scale bars in (d-i) are $100 \mathrm{~mm}$

avoid gastric corrosion, and the protective effects of the capsules were verified. After the enteric capsules entered the digestive tract, they could only be dissolved in the small intestine; in contrast, the simulated gastric fluid and other solutions were nearly insoluble (Fig. 1e). This finding demonstrated that the enteric capsules could protect MNPs from gastric acid. With the aid of 3Dprinting technology, the wearable device for losing weight could be customized according to the actual requirements (Fig. 1f). The MNPs in the small intestine would be arranged and moved in response to the magnetic force of a varying magnetic field ( $\mathrm{H}$-field), and these movements were similar to shaking. Supporting Video S1 simulates this phenomenon. Herein, a gap for magnetic field location was designed on the ventral side of the model, which could induce the vibration of the MNPs in the body. In Supporting Video S2 and Fig. S3, in vitro intestinal peristalsis is simulated, and the magnetic field strength could be controlled remotely by a mobile phone. Subsequently, the magnetic field strength at different distances from the target was studied. Theoretically, a magnetic field below $3000 \mathrm{Gs}$ is harmless to the human body ${ }^{25}$. According to the results of our study, the maximum intensity of the magnetic field was 1600 Gs, but it was enough to cause the MNPs to move (Fig. S4). This magnetic strength was too weak to make a significant difference in body health.

As the body weight of the mice decreased during the treatment period, their body length and abdomen circumference also varied. Shoulder straps similar to those of a brassiere were designed to clamp to the mouse shoulders, and the users could adjust the length of the shoulder straps according to the actual requirement (Fig. $1 \mathrm{~g})$. Moreover, PLA, the major elastic polymer component of the 3D-printing material, allowed the shape of the device to be adjusted according to the body type. Wearers could choose the usage time based on the actual food intake to realize the goal of individual therapy.

\section{Weight-loss experiment}

In this work, the C57BL/6 mice were divided into six groups. There were five different control groups, and the variables included food, $\mathrm{H}$-field, and EMNPs. All experiments were divided into two stages, each lasting 6 weeks. The first stage was a continuous dosage step, and all of the male mice were 6 weeks old ${ }^{26}$. From 7 to 12 weeks, their weights were recorded once a week. As shown in Fig. 2a, in the first 3 weeks of continued medication, both the body weight of mice that were treated with the above 
EMNPs and the variable $\mathrm{H}$-field decreased significantly, but the rest of the mice in these groups showed an upward trend in weight. The second stage was an intermittent administration phase to observe the flexibility of our treatment. The treatment was withdrawn at the 10th and 12th weeks; at the 11th week, the treatment was given. In our experiments, the body weight of the mice decreased with the treatment of EMNPs and variable $\mathrm{H}$-field. However, once the treatment was stopped, the body weight would be recovered. Furthermore, a similar experiment in SD rats was performed to ensure the effect on different species. Herein, four groups, including three typical control groups, were chosen for the experiment, with 5 rats in each group. As expected, the rats treated with EMNPs \& H-field showed weight reduction, and the others continued to gain weight (Fig. 2b). This result agreed with our previous studies. The results showed that all control groups had no visible change during the experiment, which could indicate that these factors (food, external stress, fear, etc.) have little impact on weight loss. We were also surprised to find that the mice became slimmer only if the EMNPs and variable $\mathrm{H}$-field devices worked together. For the purpose of proving our speculation of the mechanism, the peristalsis of the small intestine of the mice was observed by digital gastrointestinal contrast radiography ${ }^{27}$. The frequency of the MNPs' movements in the small intestine was approximately 90-100 times (Supporting Video S2). Supporting Video S3 (Fig. 2c) shows that the average numbers of peristalsis in $20 \mathrm{~s}$ of each group from left to right in turns were $11 \pm 2$ (group B), $13 \pm 1$ (group C), $12 \pm 1$ (group D), $11 \pm 2$ (group E), and $18 \pm 1$ (group F). This result indicated that the peristalsis of mice that had been treated with EMNPs \& H-fields was remarkably promoted compared with that of the others. The mechanism by which the EMNPs affected the metabolic process was mainly due to the motion of the magnetic nanoparticles. This movement could stimulate the peristalsis of the small intestine, speed up metabolism and reduce adsorption, finally achieving weight loss. Figure $2 \mathrm{~d}-\mathrm{i}$ shows the hematoxylin eosin (HE) staining of the tissue slices after the weight-loss experiment. The adipose cells were obviously enlarged in group B (fed with high-fat food) and had lipids in the liver, while the adipose cells and liver were normal in both group F (EMNPs \& $\mathrm{H}$-field) and group A (common food). These results showed that MNPs together with the $\mathrm{H}$-field could effectively decrease calorie intake. Figure 2j, k shows the body types of three C57BL/6 mice and SD rats, and the right one treated with EMNPs \& H-field was the thinnest. The proposed method was clearly effective in maintaining a healthy weight in the long term.

\section{TC, TG and D-xylose absorption}

To demonstrate the function of EMNPs on absorption, the levels of triglyceride (TG) and total cholesterol (TC) and the absorption of D-xylose ${ }^{28}$ in blood serum were detected after three weeks of continuous administration.

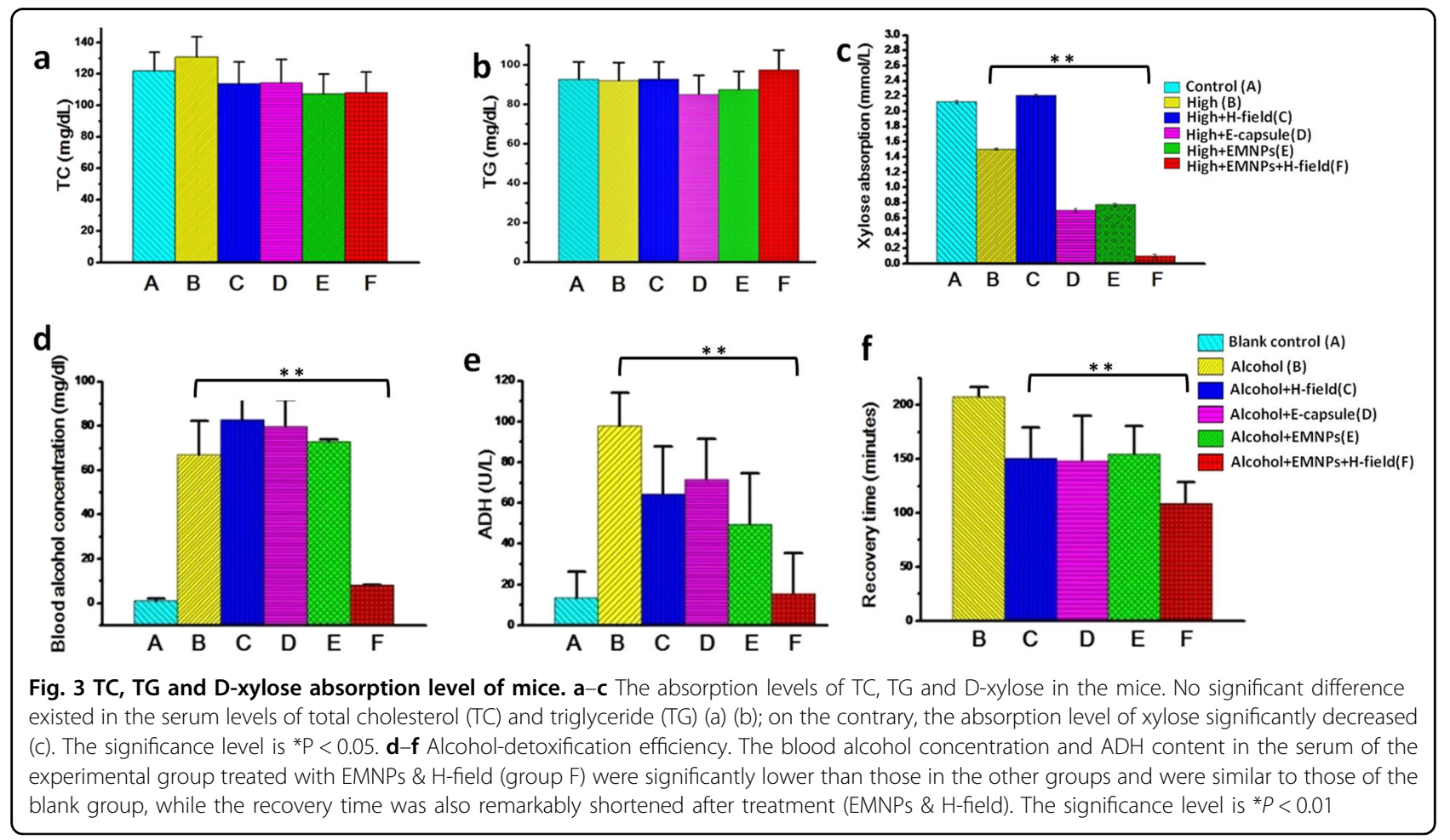




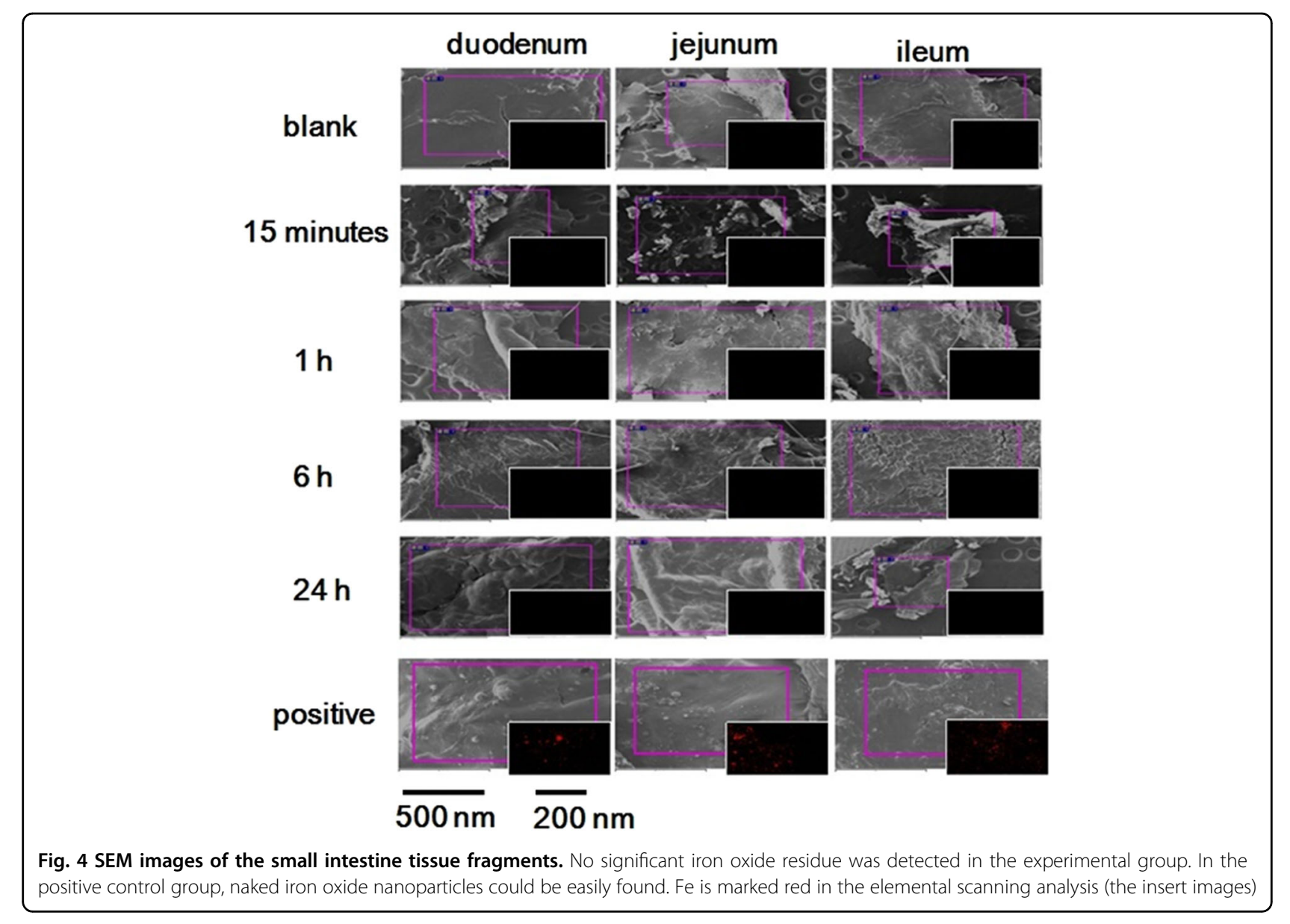

There was no significant difference in the levels of TC and TG (Fig. 3a, b), but the absorption level of D-xylose in group $\mathrm{F}$ (EMNPs \& H-field) was significantly lower than those of the other groups (Fig. 3c). In addition, group C (fed with high-fat food \& H-field) and group D (fed with high-fat food \& E-capsules) presented a relatively low absorption of xylose. This result was due to the presence of hydrophilic methylcellulose, the main component of the capsule, which could adsorb small water-soluble molecules and reduce absorption through the small intestine ${ }^{29}$. This result also indicated that the method could effectively decrease the absorption of water-soluble substances but would not form an effective barrier for fat-soluble substances. The shortcomings of lipase inhibitors could be counterbalanced, which means that the absorption of fatsoluble vitamins would not be affected, but water-soluble vitamins would be lost during the period of medication. In future practical usage, we recommend to take a vitamin complex tablet $1 \mathrm{~h}$ before the treatment. Meanwhile, we continue to work on improving the composition of the capsule, adding fat-soluble materials to trap fatty particles, and minimizing the loss of water-soluble nutrients.

\section{Alcohol detoxification of EMNPs}

Interestingly, in addition to contributing to losing weight, the EMNPs also played a role in alcohol detoxification. The blood alcohol concentration and $\mathrm{ADH}$ content in the serum increase after alcohol intoxication and have the most significant distinction in the first $90 \mathrm{~min}^{30}$. Recovery time is another persuasive index to test the effect of treatment on drunkenness. As expected, the results were extremely similar to those of the weightloss experiment (Fig. 3d, e). The blood alcohol concentration and ADH content in the serum of group F were similar to those of the blank group (group A), while the results of the other groups were almost the same as those of the alcoholism group (group B). The blood alcohol concentrations in groups $\mathrm{C}$ (H-field), D (E-capsule), and $\mathrm{E}$ (EMNPs) showed a decline compared with those in group $\mathrm{B}$, which was supposed to be caused by the hydrophilic absorbability of methylcellulose. In addition, the recovery time of group F (EMNPs \& H-field) was obviously shorter than that of the other groups. The above results of blood alcohol concentration, ADH content in the serum and recovery time showed that our method (EMNPs \& H-field) 


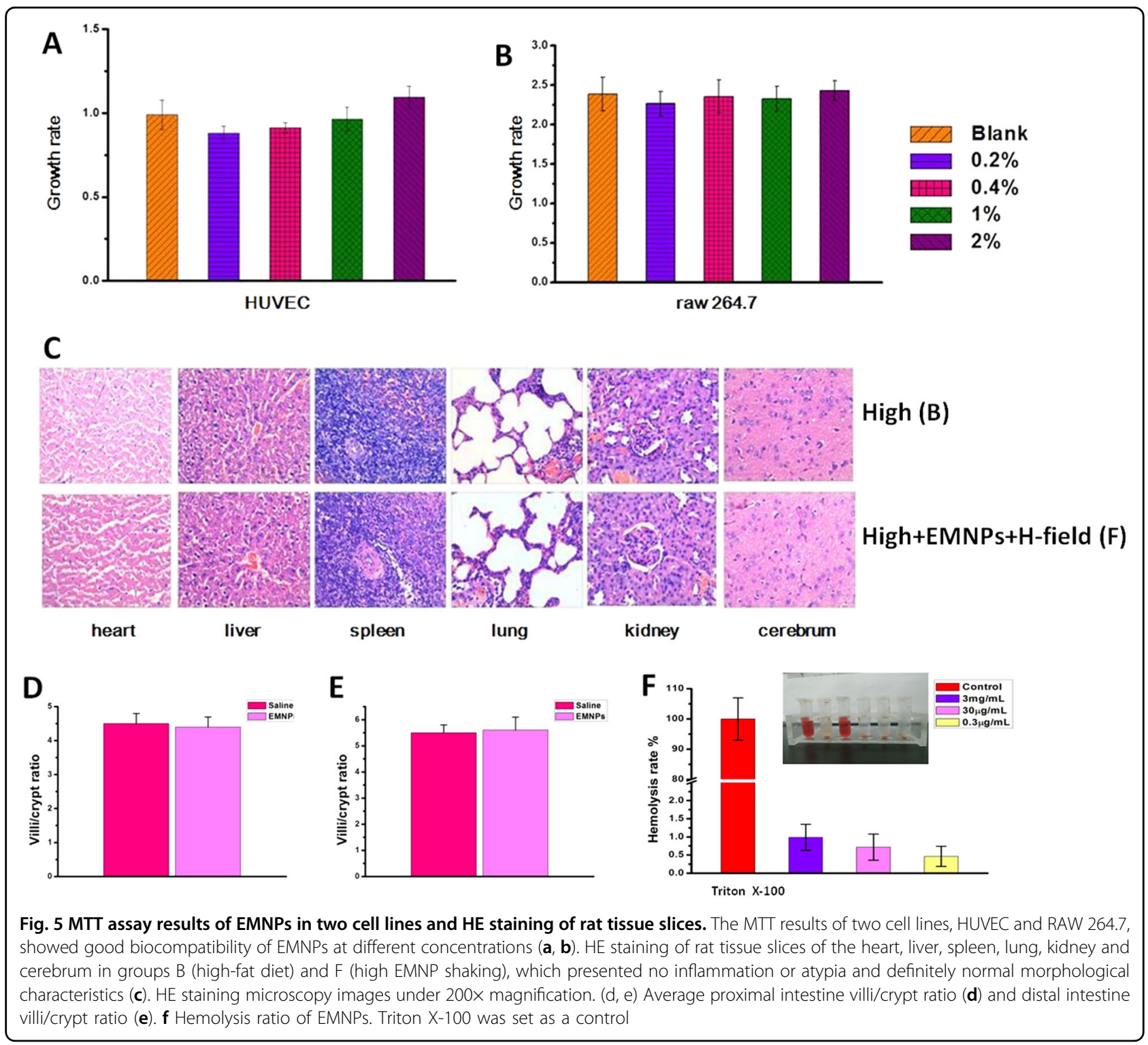

had the potential to be an antidote and a preventive measure against alcohol intoxication.

\section{Biocompatibility of MNPs}

Fe is an essential element in our body, but excess Fe in the blood would lead to deposition in the organs and would be harmful to our health. The EMNPs were supposed to attach to the mucous membrane and not the villus and would be excreted with the intestinal peristalsis. The SEM images of tissue fragments of the duodenum, jejunum and ileum were inspected to demonstrate whether MNPs were embedded in the intestine villus and absorbed into the body. The intestinal tissues were dissected at $15 \mathrm{~min}, 1 \mathrm{~h}, 6 \mathrm{~h}$ and $24 \mathrm{~h}$ after treatment. As illustrated in Fig. 5, no MNPs were detected on the surface of the small intestine tissue fragments. Similarly, the corresponding elemental scanning analysis of Fe (the insert images) indicated that after treatment of EMNPs and shaking, few MNPs attached to the mucous membrane of the small intestine regardless of time. The positive control group was treated with the same dose of drug, and the MNPs could be detected easily, as the Fe element was marked red (Fig. 4). After 6 weeks of treatment in the weight-loss experiment, the liver, renal function and lipid content were evaluated to test the safety of this new method. In Fig. S5, the results were within the normal limits of each group and were not significantly different from each other. As shown in Fig. S6, the iron element content in different organs (liver, spleen, kidney and lung) was analyzed by ICP (inductively coupled plasma) ${ }^{31}$, and we found no significant difference between the control group and the experimental group 
(EMNPs + H-field). Furthermore, no Fe peak was observed in the EDX (energy dispersive X-ray) spectra except in that of the positive control group (Fig. S7).

To evaluate the biocompatibility of the MNPs, an in vitro cytotoxicity test was performed. In Fig. 5a, b, the MTT results of two cell lines, HUVEC and RAW 264.7, show that EMNPs have no significant cytotoxicity within the effective concentration. Figure $5 \mathrm{c}$ is the HE staining of tissue slices of the heart, liver, spleen, lung, kidney and cerebrum from groups $B$ and $F$, which presented no inflammation or atypia and normal morphological characteristics. The ratio of villi/crypt in the proximal and distal intestines did not change (Fig. 5d, e), which suggested that MNPs have favorable biocompatibility with the gastrointestinal mucosa. In addition, the compatibility of the material in the blood was studied by a hemolysis test. The hemolysis rates of the MNPs were all below 1.5\%, indicating that the material has good blood compatibility (Fig. $5 f$ and Fig. S8). Overall, the SEM images and the results of the elemental scanning, elemental spectra, ICP, MTT and HE staining of the tissue slices showed that EMNPs do not adhere to the mucosa or are absorbed by the body, which ensures their safety to humans.

\section{Discussion}

Obesity could lead to a series of metabolic diseases, and the three most important pathophysiological factors are hyperglycemia, hyperlipidemia and hypertension. The proposed vibrating EMNP treatment can effectively accelerate intestinal peristalsis and reduce absorption, especially of water-soluble molecules. According to the obtained results, vibrating EMNPs can reduce the absorption of carbohydrates and lessen blood glucose accumulation and deposition in the body. This method is effective for both weight loss and alcohol detoxification; however, we still need to do further work to determine its ability to prevent hyperlipidemia and hypertension. Compared with the current drug and surgery therapy, our method was a completely physical way to minimize the amount of residual drug in the body and thus avoided several adverse effects. Furthermore, the users can adjust the dosage of EMNPs and the vibrating level themselves; additionally, 3D-printed devices can provide tunable magnetic power to achieve tailored weight reduction. Notably, because of the enteric-coated capsule membrane, most of the EMNPs could be excreted from the body after treatment. No significant difference existed in iron metabolism, and no iron signal was observed in the organs (such as the liver, spleen, kidney, and lung) of mice. Additionally, the MTT and HE staining of the tissue slices indicated in vivo safety of the proposed protocol. In brief, efficiency, flexibility, convenience and safety are the four major advantages of our protocol. Ongoing research would focus on three parts. First, we would improve the materials to minimize the loss of water-soluble vitamins and block the absorption of adipose tissue. Second, intestinal peristalsis would be stimulated by different magnetic intensities and amplitudes. Third, we would attempt to combine the size of nanoparticles with the vibration degree. Therefore, we hope that the magnetic intensity could be autonomously adjusted by selecting different motivation levels, which are marked on the devices. While both the enteric capsule (E-capsule) and magnetic nanoparticles (MNPs) have been approved by the FDA (Food and Drug Administration), we believe that the proposed method will soon be adopted in practical usage.

\section{Acknowledgements}

This work was supported by the National Natural Science Foundation of China (No. 31860263 to X.W.; No. 51102131 to F.A.; and No. 81660392 to Caifeng Xie); National Key Basic Research Program of China (2013CB531103 to H.X.); Science Foundation of Jiangxi Provincial Department of Education (KLLD14010, 20153BCB23035, 20161ACB21002, and 20165BCB19002 to X.W.); and Nanchang University Seed Grant for Biomedicine.

\section{Author details \\ ${ }^{1}$ College of Chemistry, Nanchang University, Nanchang, 330088 Jiangxi, P. R. China. ${ }^{2}$ Department of Radiation and Medical Oncology, Zhongnan Hospital, Wuhan University, Wuhan, 430071 Hubei, P. R. China. ${ }^{3}$ The Second Affiliated Hospital of Nanchang University, Nanchang, 330088 Jiangxi, P. R. China. ${ }^{4}$ The National Engineering Research Center for Bioengineering Drugs and the Technologies: Institution of Translational Medicine, Nanchang University, Nanchang, 330088 Jiangxi, P. R. China. ${ }^{5}$ Department of Chemistry, School of Chemical Engineering, Dalian University of Technology, Dalian, 116024 Liaoning, P. R. China}

\section{Author contributions}

X.L.W. conceived and designed the experiments. X.C. performed the synthesis, in vivo weight-loss experiment and blood tests. F.Y. contributed to characterization and data analysis. Y.Y.L. performed the radiography experiments. F.H. and X.X.M. accomplished the anti-intoxication studies. H.B.X. L.F.W., C.F.X. and F.R.A. revised the paper. X.L.W. is the corresponding author. All authors discussed the results and commented on the manuscript.

Conflict of interest

The authors declare that they have no conflict of interest.

\section{Publisher's note}

Springer Nature remains neutral with regard to jurisdictional claims in published maps and institutional affiliations.

Supplementary information is available for this paper at https:/doi.org/ 10.1038/s41427-019-0133-y.

Received: 17 February 2019 Revised: 29 March 2019 Accepted: 8 April 2019. Published online: 5 July 2019

\section{References}

1. Tanaka, M. et al. Macrophage-inducible C-type lectin underlies obesityinduced adipose tissue fibrosis. Nat. Commun. 5, 4982 (2014).

2. Yang, L. \& Colditz, G. A. Prevalence of overweight and obesity in the United States, 2007-2012. JAMA Intern. Med. 175, 1412-1413 (2015).

3. Lee, H. et al. A graphene-based electrochemical device with thermoresponsive microneedles for diabetes monitoring and therapy. Nat. Nanotechnol. 11, 566-572 (2016). 
4. Wu, S. L., Weng, Z. Y., Liu, X. M., Yeung, K. W. K. \& Chu, P. K. Functionalized TiO2 based nanomaterials for biomedical applications. Adv. Funct. Mater. 24 5464-5481 (2014)

5. Cai, W. et al. Oral advanced glycation endproducts (AGEs) promote insulin resistance and diabetes by depleting the antioxidant defenses AGE receptor-1 and sirtuin 1. Proc. Natl Acad. Sci. USA 109, 15888-15893 (2012).

6. Luo, N. Q. et al. Flexible piezoresistive sensor patch enabling ultralow power cuffless blood pressure measurement. Adv. Funct. Mater. 26, 1178-1187 (2016)

7. Isomaa, B. et al. Cardiovascular morbidity and mortality associated with the metabolic syndrome. Diabetes Care 24, 683-689 (2001).

8. Voytek, B. \& Knight, R. T. Prefrontal cortex and basal ganglia contributions to visual working memory. Proc. Natl Acad. Sci. USA 107, 18167-18172 (2010).

9. Xue, Y., Xu, X., Zhang, X.-Q., Farokhzad, O. C. \& Langer, R. Preventing dietinduced obesity in mice by adipose tissue transformation and angiogenesis using targeted nanoparticles. Proc. Natl Acad. Sci. USA 113 5552-5557 (2016).

10. Anonymous. Clinical guidelines on the identification, evaluation, and treatment of overweight and obesity in adults-the evidence report. National Institutes of Health. Am. J. Clin. Nutr. 68, 51S-209S (1998).

11. Padwal, RajS., Majumdar \& Sumit, R. Drug treatments for obesity: orlistat, sibutramine, and rimonabant. Lancet 369, 71-77 (2007).

12. Flynn, C. R. et al. Bile diversion to the distal small intestine has comparable metabolic benefits to bariatric surgery. Nat. Commun. 6, 7715 (2015).

13. Zoon, H. F. A. et al. Altered neural responsivity to food cues in relation to food preferences, but not appetite-related hormone concentrations after RYGBsurgery. Behav. Brain Res. 353, 194-202 (2018).

14. Garb, J., Welch, G., Zagarins, S., Kuhn, J. \& Romanelli, J. Bariatric surgery for the treatment of morbid obesity: a meta-analysis of weight loss outcomes for laparoscopic adjustable gastric banding and laparoscopic gastric bypass. Obes. Surg. 19, 1447-1455 (2009)

15. Julià, D., Gómez, N. \& Codinacazador, A. Surgical skill and complication rates after bariatric surgery. New Engl. J. Med. 370, 1223-1224 (2014).

16. Elsom, K. A., Glenn, P. M. \& Drossner, J. L. Intubation studies of the human small intestine. Am. J. Dig. Dis. 6, 593-597 (1939).
17. Wang, D. Q. Regulation of intestinal cholesterol absorption. Annu. Rev. Physiol. 69, 221-248 (2007)

18. Cole, E. T. et al. Enteric coated HPMC capsules designed to achieve intestinal targeting. Int. J. Pharm. 231, 83-95 (2002).

19. Ruiz-Hernandez, E., Baeza, A. \& Vallet-Regi, M. Smart drug delivery through dna/magnetic nanoparticle gates. ACS Nano 5, 1259-1266 (2011).

20. Eifler, A. C. \& Thaxton, C. S. Nanoparticle therapeutics: FDA approval, clinical trials, regulatory pathways, and case study. Methods Mol. Biol. 726, 325-338 (2011).

21. McNeil, N. I. The contribution of the large-intestine to energy supplies in man Am. J. Clin. Nutr. 39, 338-342 (1984).

22. Gunzerath, L., Faden, V., Zakhari, S. \& Warren, K. National Institute on Alcoho Abuse and Alcoholism report on moderate drinking. Alcohol Clin. Exp. Res. 28 829-847 (2004).

23. Leuner, C. \& Dressman, J. Improving drug solubility for oral delivery using solid dispersions. Eur. J. Pharm. Biopharm. 50, 47-60 (2000).

24. Wang, Z. L. Transmission electron microscopy of shape-controlled nanocrystals and their assemblies. J. Phys. Chem. B 104, 1153-1175 (2000).

25. Liu, Z.-C et al. Comparison of targeting distribution of two kinds of fluorouracil magnetic albumin microspheres in colorectal neoplasm nude mice in vivo under magnetic field. J. Gastrointest. Surg. 15, 622-624 (2012).

26. Zhang, $Y$. et al. Positional cloning of the mouse obese gene and its human homologue. Nature 372, 425-432 (1994).

27. Martin, T. R., Vennes, J. A., Silvis, S. E. \& Ansel, H. J. A comparison of upper gastrointestinal endoscopy and radiography. J. Clin. Gastroenterol. 2, 21-25 (1980).

28. Benson, J. A. et al. The d-xylose absorption test in malabsorption syndromes. New Engl. J. Med. 256, 335-339 (1957).

29. Lee, Y. et al. Therapeutic luminal coating of the intestine. Nat. Mater. 17 834-842 (2018).

30. Liu, Y. et al. Biomimetic enzyme nanocomplexes and their use as antidotes and preventive measures for alcohol intoxication. Nat. Nanotechnol. 8 , 187-192 (2013).

31. Hilty, F. M. et al. Iron from nanocompounds containing iron and zinc is highly bioavailable in rats without tissue accumulation. Nat. Nanotechnol. 5, 374-380 (2010). 